\title{
On Computing the Smallest Four-Coloring of Planar Graphs and Non-Self-Reducible Sets in $P^{\star}$
}

\author{
André Große $\mathrm{e}^{\mathrm{a}, 1}$ \\ a Institut für Informatik, Friedrich-Schiller-Universität Jena, 07740 Jena, Germany \\ Jörg Rothe ${ }^{\mathrm{b}, 2}$ \\ bInstitut für Informatik, Heinrich-Heine-Universität Düsseldorf, 40225 Düsseldorf, \\ Germany \\ Gerd Wechsung ${ }^{\mathrm{c}}$ \\ ${ }^{\mathrm{C}}$ Institut für Informatik, Friedrich-Schiller-Universität Jena, 07740 Jena, Germany
}

\begin{abstract}
We show that computing the lexicographically first four-coloring for planar graphs is $\Delta_{2^{-}}^{p}$ hard. This result optimally improves upon a result of Khuller and Vazirani who prove this problem NP-hard, and conclude that it is not self-reducible in the sense of Schnorr, assuming $\mathrm{P} \neq \mathrm{NP}$. We discuss this application to non-self-reducibility and provide a general related result. We also discuss when raising a problem's NP-hardness lower bound to $\Delta_{2^{-}}^{p}$ hardness can be valuable.
\end{abstract}

Key words: computational complexity, graph colorability, self-reducibility

\section{Introduction}

Khuller and Vazirani [13] proved that computing the lexicographically smallest solutions of P1-4-Color instances is NP-hard, where Pl-4-Color denotes the pla-

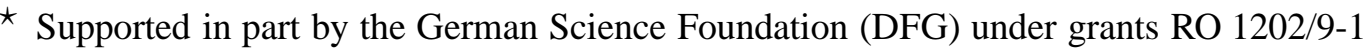
and RO 1202/9-3. A preliminary version of this paper appeared as part of [9] in the proceedings of the Seventh Italian Conference on Theoretical Computer Science.

URL: ccc.cs.uni-duesseldorf.de/ ${ }^{2}$ rothe (Jörg Rothe).

1 Current address/affiliation: Entwicklung Lasersensorik, JENOPTIK Laser, Optik, Systeme GmbH, 07745 Jena, Germany.

2 Corresponding author. Supported in part by a Heisenberg Fellowship from the DFG.
} 
nar graph four-colorability problem. They conclude that, unless $\mathrm{P}=\mathrm{NP}$, the polynomial-time decidable problem P1-4-Color is not self-reducible in the sense of Schnorr [22,23]. Noting that their result appears to be the first such non-selfreducibility result for problems in $\mathrm{P}$, they proposed as an interesting task to find other problems in $\mathrm{P}$ that are not self-reducible under some plausible assumption.

We raise Khuller and Vazirani's NP-hardness lower bound for computing the lexicographically smallest four-coloring of a planar graph to $\Delta_{2}^{p}$-hardness. Our result is optimal, since this problem belongs to (the function analog of) the class $\Delta_{2}^{p}$.

The class $\Delta_{2}^{p}=\mathrm{P}^{\mathrm{NP}}$, which belongs to the second level of the polynomial hierarchy [17,26], contains exactly the problems solvable in deterministic polynomial time with an NP oracle. Papadimitriou [18] proved that Unique-Optimal-Traveling-Salesperson is $\Delta_{2}^{p}$-complete, and Krentel [15] and Wagner [28] established many more $\Delta_{2}^{p}$-completeness results, including the result that the problem Odd-Max-SAT is $\Delta_{2}^{p}$-complete. The complexity of colorability problems has been studied in a number of papers, see, e.g., $[1,2,25,6,28,13,20]$.

As mentioned above, if for some problem in $\mathrm{P}$ computing the lexicographically smallest solution is hard, then the problem itself cannot be self-reducible in the sense of Schnorr [22,23], unless $\mathrm{P}=\mathrm{NP}$. We discuss this application to non-selfreducibility and provide a general related result. In particular, it follows from this result that even a set as simple as $\Sigma^{*}$ has representations in which it is not selfreducible in Schnorr's sense, unless $\mathrm{P}=$ NP. Finally, we conclude this paper with a discussion of when raising a problem's NP-hardness lower bound to $\Delta_{2}^{p}$-hardness can be valuable, and pose some open questions.

\section{Computing the Smallest Four-Coloring of a Planar Graph}

Appel and Haken [1,2] showed that every planar graph can be colored with no more than four colors, thus solving the famous Four Color Conjecture in the affirmative. In contrast, for each $k \geq 4$, computing the lexicographically first $k$-coloring of a planar graph is hard: Khuller and Vazirani [13] established an NP-hardness lower bound for this problem. We raise their lower bound to $\Delta_{2}^{p}$-hardness. Since the lexicographically smallest $k$-coloring of a planar graph can be computed in (the function analog of) $\Delta_{2}^{p}$, this improved lower bound is optimal.

Definition 2.1 Let $k>1$, and let $0,1, \ldots, k-1$ represent $k$ colors.

- A $k$-coloring of an undirected graph $G=(V, E)$ is a mapping $\psi_{G}: V \rightarrow$ $\{0,1, \ldots, k-1\}$.

- A k-coloring $\psi_{G}$ is said to be legal if and only if for each edge $\{u, v\} \in E$, $\psi_{G}(u) \neq \psi_{G}(v)$. 
- A graph $G$ is said to be $k$-colorable if and only if there exists a legal $k$-coloring of $G$.

- Let $\mathrm{P} 1-k$-Color denote the planar graph $k$-colorability problem.

Stockmeyer [25] proved that P1-3-Color is NP-complete, see also Garey et al. [6]. By Appel and Haken's above-mentioned result, every planar graph is fourcolorable. Thus, $\mathrm{Pl}-k$-Color is in $\mathrm{P}$ for each $k \geq 4$.

Definition 2.2 (Khuller and Vazirani [13]) Let $k>1$, and let the vertex set of a given undirected graph $G=(V, E)$ with $n$ vertices be ordered as $V=$ $\left\{v_{1}, v_{2}, \ldots, v_{n}\right\}$. Then, every $k$-coloring $\psi_{G}$ of $G$ can be represented by a string $\psi_{G}$ in $\{0,1, \ldots, k-1\}^{n}$, which is defined by $\psi_{G}=\psi_{G}\left(v_{1}\right) \psi_{G}\left(v_{2}\right) \cdots \psi_{G}\left(v_{n}\right)$.

Define the lexicographically smallest (legal) $k$-coloring by

$$
\operatorname{LF}_{\mathrm{PI}-k \text {-Color }}(G)=\min \left\{\psi_{G} \mid \psi_{G} \text { is a legal } k \text {-coloring of } G\right\} \text {, }
$$

if $G \in \mathrm{Pl}-k$-Color, where the minimum is taken with respect to the lexicographic ordering of strings, and define $\operatorname{LF}_{\mathrm{P} 1-k \text {-Color }}(G)=10^{n}$ if $G \notin \mathrm{Pl}-k$-Color.

We now prove our main result.

Theorem 2.3 Computing the lexicographically smallest $k$-coloring for planar graphs is $\Delta_{2}^{p}$-hard for any $k \geq 4$.

Proof. For simplicity, we show this claim only for $k=4$. Let $\rho_{4}$ be the reduction of Khuller and Vazirani [13, Theorem 3.1]. Recall that $\rho_{4}$ maps a given planar graph $G=(V, E)$, whose vertices are ordered as $V=\left\{v_{1}, v_{2}, \ldots, v_{m}\right\}$, to the planar graph $H=(U, F)$ defined as follows:

- The vertex set of $H$ is ordered as $U=\left\{u_{1}, u_{2}, \ldots, u_{2 m}\right\}$, where $u_{i}$ is a new vertex and $u_{m+i}=v_{i}$ is an old vertex for each $i, 1 \leq i \leq m$.

- The edge set of $H$ is defined by $F=E \cup\left\{\left\{u_{i}, u_{m+i}\right\} \mid 1 \leq i \leq m\right\}$.

It follows immediately from this construction that

(1) $G \in$ P1-3-Color $\Longleftrightarrow \operatorname{LF}_{\mathrm{Pl} \text {-4-Color }}\left(\rho_{4}(G)\right) \in\left\{0^{m} w \mid w \in\{1,2,3\}^{m}\right\}$,

that is, " $G \in \mathrm{Pl}$-3-Color?" can be decided by looking at the first $m$ bits of $\operatorname{LF}_{\mathrm{P} 1-4 \text {-Color }}(H)$.

We give a reduction from the problem Odd-Min-SAT, which is defined to be the set of all boolean formulas $F=F\left(x_{1}, x_{2}, \ldots, x_{n}\right)$ in conjunctive normal form for which, assuming $F$ is satisfiable, the lexicographically smallest satisfying assignment $\alpha:\left\{x_{1}, x_{2}, \ldots, x_{n}\right\} \rightarrow\{1,2\}$ is "odd," i.e., for which $\alpha\left(x_{n}\right)=1$. Here, "1" represents "true," and " 2 " represents "false." 
It is well known that Odd-Min-SAT is $\Delta_{2}^{p}$-complete; Krentel [15] and also Wagner [28] proved the corresponding claim for the dual problem Odd-Max-SAT.

Let $F=F\left(x_{1}, x_{2}, \ldots, x_{n}\right)$ be any given boolean formula. Without loss of generality, we may assume that $F$ is in conjunctive normal form with exactly three literals per clause. Assume that $F$ has $z$ clauses. Let $\sigma$ be the Stockmeyer reduction from 3-SAT to P1-3-Color, see Stockmeyer [25] and also Garey et al. [6]. This reduction $\sigma$, on input $F$, yields a graph $G=(V, E)$ with $m>n$ vertices, where $m=m(F)$ depends on the number $n$ of variables, the number $z$ of clauses, and the structure of $F$. Note that $F$ 's structure induces a certain number of "crossovers" of edges to guarantee the planarity of $G$; see $[6,25]$ for details.

Order the vertex set of $G$ as $V=\left\{v_{1}, v_{2}, \ldots, v_{m}\right\}$ such that

(a) for each $i, 1 \leq i \leq n, v_{i}$ represents the variable $x_{i}$, and

(b) for each $i, n<i \leq m, v_{i}$ represents some other vertex of $G$.

Note that $G$ is a planar graph satisfying the following properties:

(i) $F$ is satisfiable if and only if $G$ is 3 -colorable, using the colors 1, 2, and 3.

(ii) Every satisfying assignment $\alpha$ of $F$ corresponds to a 3-coloring $\psi_{\alpha}$ of $G$ such that for each $i, 1 \leq i \leq n, \psi_{\alpha}\left(v_{i}\right)=\alpha\left(v_{i}\right) \in\{1,2\}$. The color 3 is used for the other vertices of $G$.

Now apply the reduction $\rho_{4}$ of Khuller and Vazirani to $G$ and obtain a planar graph $H=\rho_{4}(G)=\rho_{4}(\sigma(F))$ that satisfies Equation (1) as described above. It follows immediately from this construction and from Equation (1) that

$$
\begin{aligned}
& F \in \text { Odd-Min-SAT } \Longleftrightarrow \\
& \operatorname{LF}_{\mathrm{P} 1-4-\operatorname{Col} o r}\left(\rho_{4}(\sigma(F))\right) \in\left\{0^{m} w 1 y \mid w \in\{1,2\}^{n-1} \text { and } y \in\{1,2,3\}^{m-n}\right\},
\end{aligned}
$$

that is, " $F \in$ Odd-Min-SAT?" can be decided by looking at the first $m$ bits and at the $(m+n)$ th bit of $\operatorname{LF}_{\mathrm{P} 1-4 \text {-Color }}(H)$.

For $k>4$, the claim of the theorem follows from an analogous argument that employs in place of $\rho_{4}$ the appropriate reduction $\rho_{k}$ from [13, Thm. 3.2].

\section{Non-Self-Reducible Sets in $\mathbf{P}$}

From their NP-hardness lower bound for computing the lexicographically first fourcoloring of planar graphs, Khuller and Vazirani [13] conclude that, unless $\mathrm{P}=\mathrm{NP}$, the polynomial-time decidable problem Pl-k-Color is not self-reducible for $k \geq 4$. 
The type of (functional) self-reducibility used by Khuller and Vazirani is due to Schnorr [22,23], see also [5]. For more background on self-reducibility, see, e.g., $[24,12,21]$.

\section{Definition 3.1 (Schnorr [22,23])}

- Let $\Sigma$ and $\Gamma$ be alphabets with at least two symbols each. Instances of problems are encoded over $\Sigma$, and solutions of problems are encoded over $\Gamma$. For any set $B \subseteq \Sigma^{*} \times \Gamma^{*}$ and any polynomial $p$, the p-projection of $B$ is defined to be the set

$$
\operatorname{proj}_{p}(B)=\left\{x \in \Sigma^{*} \mid\left(\exists y \in \Gamma^{*}\right)[|y| \leq p(|x|) \text { and }(x, y) \in B]\right\}
$$

If $A=\operatorname{proj}_{p}(B)$, we say $A$ has the representation $(B, p)$.

- A partial order $\leq$ on $\Sigma^{*}$ is polynomially well-founded and length-bounded if and only if there exists a polynomial $q$ such that

(a) every $\leq$-decreasing chain with maximum element $x$ has at most $q(|x|)$ elements, and

(b) for all strings $x, y \in \Sigma^{*}, x<y$ implies $|x| \leq q(|y|)$.

- Let $A=\operatorname{proj}_{p}(B)$ for some set $B \subseteq \Sigma^{*} \times \Gamma^{*}$ and some polynomial $p$. The projection $A$ is said to be self-reducible with respect to its representation $(B, p)$ if and only if there exist a polynomial-time computable function $g$ mapping from $\Sigma^{*} \times \Gamma$ to $\Sigma^{*}$ and a polynomially well-founded and length-bounded partial order $\leq$ such that for all strings $x \in \Sigma^{*}$, for all strings $y \in \Gamma^{*}$, and for all symbols $\gamma \in \Gamma$

(i) $g(x, \gamma)<x$, and

(ii) $(x, \gamma y) \in B \Longleftrightarrow(g(x, \gamma), y) \in B$.

If the representation $(B, p)$ of $A=\operatorname{proj}_{p}(B)$ is clear from the context, we omit the phrase "with respect to its representation $(B, p)$."

We mention in passing that various other important types of self-reducibility have been studied, such as the self-reducibility defined by Meyer and Paterson [16] and the disjunctive self-reducibility studied by Selman [24], Ko [14], and many others. We refer the reader to the excellent survey by Joseph and Young [12] for an overview and for pointers to the literature. Note that, in sharp contrast with Schnorr's self-reducibility, every set in P is self-reducible in the sense of Meyer and Paterson [16], Ko [14], and Selman [24].

Definition 3.2 Let $\Sigma=\{0,1\}$. Given any set $A$ in NP with $A \subseteq \Sigma^{*}$, there is an associated set $B_{A} \subseteq \Sigma^{*} \times \Sigma^{*}$ and an associated polynomial $p_{A}$ such that $B_{A}$ is in $\mathrm{P}$ and $A=\operatorname{proj}_{p_{A}}\left(B_{A}\right)$.

- For any $x \in \Sigma^{*}$, define the set of solutions for $x$ with respect to $B_{A}$ and $p_{A}$ by

$$
\operatorname{Sol}_{\left(B_{A}, p_{A}\right)}(x)=\left\{y \in \Sigma^{*}|| y \mid \leq p_{A}(|x|) \text { and }(x, y) \in B_{A}\right\} \text {. }
$$

Note that $x \in A$ if and only if $\operatorname{Sol}_{\left(B_{A}, p_{A}\right)}(x) \neq \emptyset$. 
- For any $x \in \Sigma^{*}$, define the lexicographically first solution with respect to $B_{A}$ and $p_{A}$ by

$$
\operatorname{LF}_{\left(B_{A}, p_{A}\right)}(x)= \begin{cases}\min \operatorname{Sol}_{\left(B_{A}, p_{A}\right)}(x) & \text { if } x \in A \\ \operatorname{bin}\left(2^{p(|x|)}\right) & \text { otherwise, }\end{cases}
$$

where the minimum is taken with respect to the lexicographic ordering of $\Sigma^{*}$, and $\operatorname{bin}(n)$ denotes the binary representation of the integer $n$ without leading zeros.

If the representation $\left(B_{A}, p_{A}\right)$ of $A=\operatorname{proj}_{p_{A}}\left(B_{A}\right)$ is clear from the context, we use $\operatorname{Sol}_{A}(x)$ and $\mathrm{LF}_{A}(x)$ as shorthands for, respectively, $\operatorname{Sol}_{\left(B_{A}, p_{A}\right)}(x)$ and $\operatorname{LF}_{\left(B_{A}, p_{A}\right)}(x)$.

It is well known that if $A$ is self-reducible then $\mathrm{LF}_{A}$ can be computed in polynomial time by prefix search, via suitable queries to the oracle $A$. Moreover, if $A$ is in $\mathrm{P}$ then $\mathrm{LF}_{A}$ can even be computed in polynomial time without any oracle queries. It follows that if $A$ is in $\mathrm{P}$ yet computing $\mathrm{LF}_{A}$ is NP-hard then $A$ cannot be selfreducible, assuming $\mathrm{P} \neq \mathrm{NP}$.

Khuller and Vazirani [13] propose to find polynomial-time decidable problems other than $\mathrm{P} 1-4-C o l o r$ that are non-self-reducible, under the assumption $\mathrm{P} \neq \mathrm{NP}$. Theorem 3.5 below provides a general result showing that it is almost trivial to find such problems: For any NP problem $A$ for which $\mathrm{LF}_{A}$ is hard to compute, one can define a $\mathrm{P}$-decidable version $D$ of $A$ such that $\mathrm{LF}_{D}$ is still hard to compute; hence, $D$ is not self-reducible, assuming $\mathrm{P} \neq \mathrm{NP}$.

To formulate this result, we now define the functional many-one reducibility that was introduced by Vollmer [27] as a potentially stricter reducibility notion than Krentel's metric reducibility [15]. We also define the function class $\min \cdot P$ that was introduced by Hempel and Wechsung [11].

Definition 3.3 (Vollmer [27]) Let $f$ and $h$ be functions from $\Sigma^{*}$ to $\Sigma^{*}$.

- We say that $f$ is polynomial-time functionally many-one reducible to $h$ (in symbols, $f \leq_{\mathrm{m}}^{\mathrm{FP}} h$ ) if and only if there exists a polynomial-time computable function $g$ such that for all $x \in \Sigma^{*}, f(x)=h(g(x))$.

- We say that $h$ is $\leq_{\mathrm{m}}^{\mathrm{FP}}$-hard for a function class $\mathcal{C}$ if and only if for every $f \in \mathcal{C}$, $f \leq_{\mathrm{m}}^{\mathrm{FP}} h$.

- We say that $h$ is $\leq_{\mathrm{m}}^{\mathrm{FP}}$-complete for $\mathcal{C}$ if and only if $h \in \mathcal{C}$ and $h$ is $\leq_{\mathrm{m}}^{\mathrm{FP}}$-hard.

Definition 3.4 (Hempel and Wechsung [11]) Define the class min $\cdot \mathrm{P}$ to consist of all functions $f$ for which there exist a set $A \in \mathrm{P}$ and a polynomial $p$ such that for all $x \in \Sigma^{*}$,

$$
f(x)=\min \left\{y \in\{0,1\}^{*}|| y \mid \leq p(|x|) \text { and }(x, y) \in A\right\},
$$

where $(\cdot, \cdot): \Sigma^{*} \times \Sigma^{*} \rightarrow \Sigma^{*}$ is a standard pairing function. If the set over which the minimum is taken is empty, define by convention $f(x)=\operatorname{bin}\left(2^{p(|x|)}\right)$. 
Note that $\mathrm{LF}_{A}=\mathrm{LF}_{(B, p)}$ is in min $\cdot \mathrm{P}$ for every NP set $A$ and for every representation of $A$ as a $p$-projection $A=\operatorname{proj}_{p}(B)$ of some suitable set $B \in \mathrm{P}$ and polynomial $p$.

Theorem 3.5 Let $\Sigma=\{0,1\}$, let $A \subseteq \Sigma^{*}$ be any set in $\mathrm{NP}$, let $B \subseteq \Sigma^{*} \times \Sigma^{*}$ and $D \subseteq \Sigma^{*}$ be sets in $\mathrm{P}$, and let $p$ be a polynomial such that $A=\operatorname{proj}_{p}(B) \subseteq D$ and $\mathrm{LF}_{A}$ is $\leq_{\mathrm{m}}^{\mathrm{FP}}$-complete for min $\cdot \mathrm{P}$. Then, there exist a set $C \subseteq \Sigma^{*} \times \Sigma^{*}$ in $\mathrm{P}$ and a polynomial $q$ such that $D=\operatorname{proj}_{q}(C)$ and computing $\mathrm{LF}_{D}$ is $\Delta_{2}^{p}$-hard.

Hence, $D$ is not self-reducible with respect to $(C, q)$, assuming $\mathrm{P} \neq \mathrm{NP}$.

Proof. Define the set

$$
C=(B \cap\{(x, y)|| y \mid \leq p(|x|)\}) \cup\left\{\left(x, \operatorname{bin}\left(2^{p(|x|)}\right)\right) \mid x \in D\right\},
$$

and let $q(n)=p(n)+1$ for all $n$. Note that $C \in \mathrm{P}$ and $D=\operatorname{proj}_{q}(C)$. It also follows that $\operatorname{LF}_{A}(x) \equiv \operatorname{LF}_{D}(x) \bmod 2$ if $x \in D$, and $\operatorname{LF}_{A}(x) \equiv \operatorname{LF}_{D}(x) \equiv 0$ mod 2 if $x \notin D$. Thus, for all $x, \mathrm{LF}_{A}(x) \equiv \mathrm{LF}_{D}(x) \bmod 2$.

We now show that computing $\mathrm{LF}_{D}$ is as hard as deciding the $\Delta_{2}^{p}$-complete problem Odd-Min-SAT, which was defined in Section 2. Since $\mathrm{LF}_{A}$ is $\leq_{\mathrm{m}}^{\mathrm{FP}}$-complete for min $\cdot \mathrm{P}$, we have $\operatorname{LF}_{\mathrm{SAT}}(F)=\mathrm{LF}_{A}(t(F))$ for some polynomial-time computable function $t$. Hence,

$$
\begin{aligned}
F \in \text { Odd-Min-SAT } & \Longleftrightarrow \operatorname{LF}_{\mathrm{SAT}}(F) \equiv 1 \bmod 2 \\
& \Longleftrightarrow \operatorname{LF}_{A}(t(F)) \equiv 1 \bmod 2 \\
& \Longleftrightarrow \operatorname{LF}_{D}(t(F)) \equiv 1 \bmod 2 .
\end{aligned}
$$

Thus, one can decide whether or not $F$ belongs to Odd-Min-SAT by looking at the last bit of $\mathrm{LF}_{D}(t(F))$.

Corollary 3.6 If $\mathrm{P} \neq \mathrm{NP}$ then $\Sigma^{*}$ has representations in which it is not selfreducible.

Proof. Replacing the set $D$ of Theorem 3.5 by $\Sigma^{*}$, it is clear that the hypothesis of the theorem can be satisfied by suitably choosing $A, B$, and $p .^{3}$ It follows that $\Sigma^{*}$, unconditionally, has representations in which it is not self-reducible in the sense of Schnorr, unless $\mathrm{P}=\mathrm{NP}$.

\footnotetext{
${ }^{3}$ Concrete examples of $A, B$, and $p$ are given in Section 4 , where we assume that the problem $A$ (e.g., $A=\mathrm{P}$-SAT) as well as the set of solutions for instances of $A$ are suitably encoded over $\Sigma$. Thus $A \subseteq \Sigma^{*}=D$ and $B \subseteq \Sigma^{*} \times \Sigma^{*}$.
} 


\section{Conclusions and Open Questions}

In Theorem 2.3, we strengthened Khuller and Vazirani's [13] lower bound for computing the lexicographically first four-coloring for planar graphs from NP-hardness to $\Delta_{2}^{p}$-hardness. The non-self-reducibility of the P1-4-Color problem follows immediately from these lower bounds.

Since $\mathrm{P} \neq \mathrm{NP}$ is equivalent to $\mathrm{P} \neq \Delta_{2}^{p}$, our strengthened lower bound for computing $\mathrm{LF}_{\mathrm{P} 1-k \text {-Color }}(G)$ from Theorem 2.3 does not give strengthened evidence regarding the non-self-reducibility of Pl- $k$-Color. However, raising a problem's lower bound so as to match its upper bound is important in its own right.

In addition, we now give another reason of why this improved lower bound may be valuable, by re-iterating a point that has first been made by Hemaspaandra et al. [10], who discuss the issue of why and when it may be valuable to raise a problem's NP-hardness lower bound to $\Theta_{2}^{p}$-hardness, with regard to other computational models such as one-sided error randomized polynomial time or unambiguous polynomial time. Just as $\Delta_{2}^{p}$, the class $\Theta_{2}^{p}=\mathrm{P}^{\mathrm{NP}}[\mathrm{log}]$ belongs to the second level of the polynomial hierarchy; note that $\mathrm{NP} \subseteq \Theta_{2}^{p} \subseteq \Delta_{2}^{p} \subseteq \mathrm{NP}^{\mathrm{NP}}$. Rephrasing for the class $\Delta_{2}^{p}$ a question that Hemaspaandra et al. [10] studied for $\Theta_{2}^{p}$, we ask: Given a complexity class $\mathcal{C}$, is it currently known that NP $\subseteq \mathcal{C}$ if and only if $\Delta_{2}^{p} \subseteq \mathcal{C}$ ? The answer to this question is the key to the issue of whether or not raising an NPhardness lower bound to $\Delta_{2}^{p}$-hardness indeed may have some value: If the answer is yes, then the raised lower bound is worthless with respect to the computational model captured by $\mathcal{C}$; if the answer is no, then the raised lower bound may potentially be valuable for $\mathcal{C}$.

Table 1 provides some answers to the above question for various classes $\mathcal{C}$ with respect to $\Delta_{2}^{p}$-hardness. ${ }^{4}$ In most cases (namely, for $\mathcal{C}$ being one of $\mathrm{P}, \mathrm{BPP}, \mathrm{RP}$, $\mathrm{ZPP}$, and UP), the answer for $\Delta_{2}^{p}$ is the same as for $\Theta_{2}^{p}$, by essentially the argument given in [10]. However, if $\mathcal{C}$ is either PP or GP, the answer for $\Delta_{2}^{p}$ differs from that for $\Theta_{2}^{p}$. In particular, since NP $\subseteq$ PP and $\Theta_{2}^{p} \subseteq \mathrm{PP}$ (see [4]), raising NP-hardess to $\Theta_{2}^{p}$-hardness is worthless for PP. In contrast, raising NP-hardess to $\Delta_{2}^{p}$-hardness may potentially be valuable for PP, since it is not known whether $\Delta_{2}^{p} \subseteq \mathrm{PP}$; there is even a relativized counterexample for $\Delta_{2}^{p} \subseteq \mathrm{PP}$ (see [3]). And for $\mathcal{C}=G \mathrm{~F}$, the closure properties of GP imply that raising NP-hardess to $\Theta_{2}^{p}$-hardness is worthless (see [10]), but do not seem to suffice in any obvious way to yield the same claim for $\Delta_{2}^{p}$-hardness. Again, there are relativized counterexamples for the inclusion $\Delta_{2}^{p} \subseteq$ GP - and even relativizations that separate the entire polynomial hierarchy from GP with immunity, see [19]. However, unlike for PP, these relativized separations do not give us any more insight regarding the value of raising NP-hardness to $\Delta_{2^{-}}^{p}$ hardness for $\mathrm{FP}$, since $\mathrm{NP} \subseteq \mathrm{GP}$ is not known to hold either.

\footnotetext{
$\overline{{ }^{4}}$ For the definitions of the classes $\mathcal{C}$ discussed in Table 1, the reader is referred to [10] and the original literature cited therein.
} 


\begin{tabular}{||l|c||c|c||}
\hline \hline Computational Model & $\mathcal{C}$ & $\mathrm{NP} \subseteq \mathcal{C} \Longleftrightarrow \Delta_{2}^{p} \subseteq \mathcal{C} ?$ & Reference \\
\hline \hline Deterministic Polynomial Time & $\mathrm{P}$ & yes & {$[17,26]$} \\
\hline Probabilistic Polynomial Time & $\mathrm{PP}$ & not known & but see [3] \\
\hline $\begin{array}{l}\text { Bounded-Error } \\
\text { Probabilistic Polynomial Time }\end{array}$ & $\mathrm{BPP}$ & yes & {$[7,29]$} \\
\hline $\begin{array}{l}\text { Zero-Error } \\
\text { Probabilistic Polynomial Time }\end{array}$ & $\mathrm{ZPP}$ & yes & {$[7]$} \\
\hline Random Polynomial Time & $\mathrm{RP}$ & not known & but see [10] \\
\hline Exact Counting & $\mathrm{GP}$ & not known & but see [10] \\
\hline Unambiguous Polynomial Time & $\mathrm{UP}$ & not known & but see [10] \\
\hline \hline
\end{tabular}

Table 1

When can it be useful to raise NP-hardness to $\Delta_{2}^{p}$-hardness?

Khuller and Vazirani [13] asked whether similar non-self-reducibility results can be proven for problems in $\mathrm{P}$ other than $\mathrm{P} 1-4-\mathrm{Col}$ or, under some plausible assumption such as $\mathrm{P} \neq \mathrm{NP}$. We established as Theorem 3.5 a general result showing that it is almost trivial to find such problems.

This general result subsumes a number of results [8] providing concrete-although somewhat artificial—problems in P that are not self-reducible in Schnorr's sense, unless $\mathrm{P}=\mathrm{NP}$. Why are these problems artificial? The reason is that they are $\mathrm{P}$ versions of standard NP-complete problems — such as the satisfiability problem, the clique problem, and the knapsack problem - that are defined by

(a) encoding directly into each solvable problem instance a trivial solution to this instance, and simultaneously

(b) ensuring that computing the smallest solution remains a hard problem by fixing a suitable ordering of the solutions to a given problem instance.

Here are some examples of such problems:

(1) (a) P-SAT is the set of pairs $\left(F, x_{i}\right)$ such that $F$ is a boolean formula in conjunctive normal form and $x_{i}$ is a variable occurring in each clause of $F$ in positive form.

(b) Let the variables of a given formula $F$ be ordered as $F=$ $F\left(x_{1}, x_{2}, \ldots, x_{n}\right)$. Just as for the satisfiability problem, a solution to $a$ P-SAT instance $I=\left(F, x_{i}\right)$ is any satisfying assignment $\psi_{I}$ of $F$. A solution $\psi_{I}$ of $I$ is represented by the string $\psi_{I}$ in $\{0,1\}^{n}$ that is defined by $\psi_{I}=\psi_{I}\left(x_{1}\right) \psi_{I}\left(x_{2}\right) \cdots \psi_{I}\left(x_{n}\right)$, where "1" represents "true" and " 0 " represents "false."

(2) (a) P-Clique is the set of pairs $(G, C)$ such that $G=(V, E)$ is a graph and 
$C \subseteq V$ is a clique in $G$.

(b) Let the vertex set of a given graph $G=(V, E)$ be ordered as $V=$ $\left\{v_{1}, v_{2}, \ldots, v_{n}\right\}$. Just as for the clique problem, a solution to a P-Clique instance $I=(G, C)$ is any clique $\hat{C} \subseteq V$ that is of size at least $\|C\|$. A solution $\hat{C}$ of $I$ is represented by the string $\psi_{I}$ in $\{0,1\}^{n}$ that is defined by $\psi_{I}=\chi_{\hat{C}}\left(v_{1}\right) \chi_{\hat{C}}\left(v_{2}\right) \cdots \chi_{\hat{C}}\left(v_{n}\right)$, where $\chi_{\hat{C}}$ denotes the characteristic function of $\hat{C}$, i.e., $\chi_{\hat{C}}(v)=1$ if $v \in \hat{C}$, and $\chi_{\hat{C}}(v)=0$ if $v \notin \hat{C}$.

(3) (a) P-Knapsack is the set of tuples $(U, s, v, k, b)$ such that $U$ is a finite set, $s$ and $v$ are functions mapping from $U$ to the positive integers, and there exists an element $u \in U$ satisfying $s(u) \leq b$ and $v(u) \geq k$.

(b) Let the set $U$ of a given P-Knapsack instance $I=(U, s, v, k, b)$ be ordered as $U=\left\{u_{1}, u_{2}, \ldots, u_{n}\right\}$. Just as for the knapsack problem, a solution to $I$ is any subset $\hat{U} \subseteq U$ that satisfies the "knapsack property," i.e., that satisfies the conditions

$$
\sum_{u \in \hat{U}} s(u) \leq b \text { and } \sum_{u \in \hat{U}} v(u) \geq k .
$$

A solution $\hat{U}$ of $I$ is represented by the string $\psi_{I}$ in $\{0,1\}^{n}$ that is defined by $\psi_{I}=\chi_{\hat{U}}\left(v_{1}\right) \chi_{\hat{U}}\left(v_{2}\right) \cdots \chi_{\hat{U}}\left(v_{n}\right)$.

Note that the lexicographic ordering of strings induces a suitable ordering of the solutions to a given problem instance. For each of the $\mathrm{P}$ problems $\Pi$ defined above, computing $\mathrm{LF}_{\Pi}$ can be shown to be NP-hard [8], which implies that $\Pi$ is non-selfreducible unless $\mathrm{P}=\mathrm{NP}$.

Analogously, every standard NP-complete problem yields such an artificial, nonself-reducible problem in $\mathrm{P}$. In contrast, the $\mathrm{Pl}-4$-Color problem is a quite natural problem. Is it possible to prove, under a plausible assumption such as $\mathrm{P} \neq \mathrm{NP}$, the non-self-reducibility of other natural problems in $\mathrm{P}$ ?

Acknowledgments. We thank the anonymous referees of the conference and journal versions of this paper for their helpful and insightful comments. In particular, we thank the referee who suggested an idea that led to Theorem 3.5, which subsumes some results from an earlier draft of this paper. We very much appreciate this paper's prompt handling by the editor, Lane A. Hemaspaandra.

\section{References}

[1] K. Appel and W. Haken. Every planar map is 4-colorable - 1: Discharging. Illinois J. Math, 21:429-490, 1977.

[2] K. Appel and W. Haken. Every planar map is 4-colorable - 2: Reducibility. Illinois J. Math, 21:491-567, 1977. 
[3] R. Beigel. Perceptrons, PP, and the polynomial hierarchy. Computational Complexity, 4(4):339-349, 1994.

[4] R. Beigel, L. Hemachandra, and G. Wechsung. Probabilistic polynomial time is closed under parity reductions. Information Processing Letters, 37(2):91-94, 1991.

[5] A. Borodin and A. Demers. Some comments on functional self-reducibility and the NP hierarchy. Technical Report TR 76-284, Cornell Department of Computer Science, Ithaca, NY, July 1976.

[6] M. Garey, D. Johnson, and L. Stockmeyer. Some simplified NP-complete graph problems. Theoretical Computer Science, 1:237-267, 1976.

[7] J. Gill. Computational complexity of probabilistic Turing machines. SIAM Journal on Computing, 6(4):675-695, 1977.

[8] A. Große. Partielle Lösungen NP-vollständiger Probleme. Diploma thesis, FriedrichSchiller-Universität Jena, Institut für Informatik, Jena, Germany, December 1999. In German.

[9] A. Große, J. Rothe, and G. Wechsung. Relating partial and complete solutions and the complexity of computing smallest solutions. In Proceedings of the Seventh Italian Conference on Theoretical Computer Science, pages 339-356. Springer-Verlag Lecture Notes in Computer Science \#2202, October 2001.

[10] E. Hemaspaandra, L. Hemaspaandra, and J. Rothe. Raising NP lower bounds to parallel NP lower bounds. SIGACT News, 28(2):2-13, June 1997.

[11] H. Hempel and G. Wechsung. The operators min and max on the polynomial hierarchy. International Journal of Foundations of Computer Science, 11(2):315-342, 2000.

[12] D. Joseph and P. Young. Self-reducibility: Effects of internal structure on computational complexity. In A. Selman, editor, Complexity Theory Retrospective, pages 82-107. Springer-Verlag, 1990.

[13] S. Khuller and V. Vazirani. Planar graph coloring is not self-reducible, assuming P $\neq$ NP. Theoretical Computer Science, 88(1):183-189, 1991.

[14] K. Ko. On self-reducibility and weak P-selectivity. Journal of Computer and System Sciences, 26(2):209-221, 1983.

[15] M. Krentel. The complexity of optimization problems. Journal of Computer and System Sciences, 36:490-509, 1988.

[16] A. Meyer and M. Paterson. With what frequency are apparently intractable problems difficult? Technical Report MIT/LCS/TM-126, MIT Laboratory for Computer Science, Cambridge, MA, 1979.

[17] A. Meyer and L. Stockmeyer. The equivalence problem for regular expressions with squaring requires exponential space. In Proceedings of the 13th IEEE Symposium on Switching and Automata Theory, pages 125-129, 1972.

[18] C. Papadimitriou. On the complexity of unique solutions. Journal of the ACM, 31(2):392-400, 1984. 
[19] J. Rothe. Immunity and simplicity for exact counting and other counting classes. R.A.I.R.O. Theoretical Informatics and Applications, 33(2):159-176, March/April 1999.

[20] J. Rothe. Exact complexity of Exact-Four-Colorability. Information Processing Letters, 87(1):7-12, July 2003.

[21] J. Rothe. Complexity Theory and Cryptology. An Introduction to Cryptocomplexity. EATCS Texts in Theoretical Computer Science. Springer-Verlag, Berlin, Heidelberg, New York, 2005.

[22] C. Schnorr. Optimal algorithms for self-reducible problems. In S. Michaelson and R. Milner, editors, Proceedings of the 3rd International Colloquium on Automata, Languages, and Programming, pages 322-337, University of Edinburgh, July 1976. Edinburgh University Press.

[23] C. Schnorr. On self-transformable combinatorial problems, 1979. Presented at IEEE Symposium on Information Theory, Udine, and Symposium über Mathematische Optimierung, Oberwolfach.

[24] A. Selman. Natural self-reducible sets. SIAM Journal on Computing, 17(5):989-996, 1988.

[25] L. Stockmeyer. Planar 3-colorability is NP-complete. SIGACT News, 5(3):19-25, 1973.

[26] L. Stockmeyer. The polynomial-time hierarchy. Theoretical Computer Science, 3(1):1-22, 1977.

[27] H. Vollmer. On different reducibility notions for function classes. In Proceedings of the 11th Annual Symposium on Theoretical Aspects of Computer Science, pages 449-460. Springer-Verlag Lecture Notes in Computer Science \#775, February 1994.

[28] K. Wagner. More complicated questions about maxima and minima, and some closures of NP. Theoretical Computer Science, 51:53-80, 1987.

[29] S. Zachos and H. Heller. A decisive characterization of BPP. Information and Control, 69:125-135, 1986. 